\title{
Monte Carlo simulation for the Touschek effect with the crab-waist scheme
}

\author{
M. Boscolo* and P. Raimondi \\ INFN/LNF, Via Enrico Fermi 40, 00044 Frascati (Roma), Italy
}

(Received 10 April 2012; published 11 October 2012)

\begin{abstract}
The Touschek effect is one of the dominant loss mechanisms for lepton storage rings of low emittance (i.e. high bunch density) and low or moderate beam energy. So, in the past years, for all lepton circular machines, either colliders or modern synchrotron light sources, either due to high bunch density or to beam energy, more and more efforts have been made for a comprehensive evaluation of this effect. This paper describes the simulation tool developed to evaluate the actions relevant to minimize the Touschek effect, such as position and shape of scrapers and detector shieldings, interaction region optics design with a particular attention to sextupoles for their relevant nonlinear effects. Relevant results obtained through the years at the low energy $\mathrm{e}^{+} \mathrm{e}^{-}$collider DAфNE are presented. A reduction of the luminosity normalized to the background of more than 1 order of magnitude is obtained with an increase in luminosity from $10^{31}$ to $10^{32} \mathrm{~cm}^{-2} \mathrm{~s}^{-1}$ and a small decrease in lifetime. The corresponding data Monte Carlo simulation comparison for each action taken is presented, showing an overall good agreement.
\end{abstract}

DOI: 10.1103/PhysRevSTAB.15.104201

PACS numbers: 29.20. $-\mathrm{c}, 29.27 .-\mathrm{a}, 41.75 .-\mathrm{i}$

\section{INTRODUCTION}

The Touschek effect is strong when beams are dense at relatively low energy. The Coulomb scattering between particles in a stored bunch induces an energy exchange between transverse and longitudinal motions. In this process, small transverse momentum fluctuations are transformed into magnified longitudinal fluctuations because of the relativistic Lorentz factor in the transformation. Offmomentum particles can exceed the radio-frequency (rf) momentum acceptance, or they may hit the aperture when displaced by dispersion. In both cases they get lost. The effect was first observed in the storage ring AdA and Bruno Touschek explained it, hence the name Touschek scattering [1].

The loss rate in this process is given by

$$
d N_{b} / d t=-\alpha N_{b}^{2},
$$

where $N_{b}$ is the number of electrons per bunch and assuming that other parameters (i.e. bunch length) are constant. From relation (1) we get the number of particles per bunch after the time $t$ :

$$
N_{b}=N_{0} /\left(1+\alpha N_{b} t\right)
$$

The half-life $\tau$, known as the Touschek lifetime, is given by

$$
\tau=-N_{0} / \dot{N}_{0}
$$

\footnotetext{
*Corresponding author. manuela.boscolo@lnf.infn.it
}

Published by the American Physical Society under the terms of the Creative Commons Attribution 3.0 License. Further distribution of this work must maintain attribution to the author(s) and the published article's title, journal citation, and DOI.
The analysis used to explain the results obtained in AdA (see Bruck textbook [2]) is based on the further approximation that the transverse momentum is nonrelativistic. Bruck's result is as follows:

$$
\frac{1}{\tau}=\frac{\sqrt{\pi} r_{e}^{2} c N}{\gamma^{3}(4 \pi)^{3 / 2} V \sigma_{x}^{\prime} \varepsilon^{2}} C\left(u_{\min }\right)
$$

where $r_{e}$ is the classical electron radius; $c$ is the speed of light; $\gamma=E / m_{e} c^{2}$, where $E$ is the electron energy in the laboratory system and $m_{e}$ the electron rest mass; $V$ is the bunch volume $V=\sigma_{x} \sigma_{y} \sigma_{z} ; \varepsilon=\Delta E / E$ is the maximum relative energy deviation; $\sigma_{x}^{\prime}=\sqrt{\frac{\varepsilon_{x}}{\beta}+\sigma_{p}^{2}\left(D_{x}^{\prime}+D_{x} \frac{\alpha_{x}}{\beta_{x}}\right)^{2}}$; $u_{\min }=\left(\frac{\varepsilon}{\gamma \sigma_{x}^{\prime}}\right)^{2}$ and the slowing varying function $C\left(u_{\min }\right)$ is given by

$C\left(u_{\min }\right)=\int_{u_{\min }}^{\infty} \frac{1}{u^{2}}\left[u-u_{\min }-\frac{1}{2} \ln \left(\frac{u}{u_{\min }}\right)\right] e^{-u} d u$.

For $u_{\min }<0.01$, Bruck gives the approximation $C\left(u_{\min }\right)=\ln \left(1 / 1.78 u_{\min }\right)-1.5$, otherwise it is evaluated by a parametrized formula very close to the exact one (5).

The approximated formula is plotted together with the exact and parametrized ones in Fig. 1. The approximation is valid almost everywhere for DAфNE [3], being $u_{\text {min }}$ small.

From Bruck's formula (4), simple scaling laws from beam parameters connected to bunch density can be derived, thus having an immediate feeling about the Touschek effect behavior. First of all, the Touschek effect scales as the third power of the energy, $1 / \tau \sim \gamma^{3}$. Consequently, high energy lepton machines are almost insensitive to this effect, while new generation lepton 


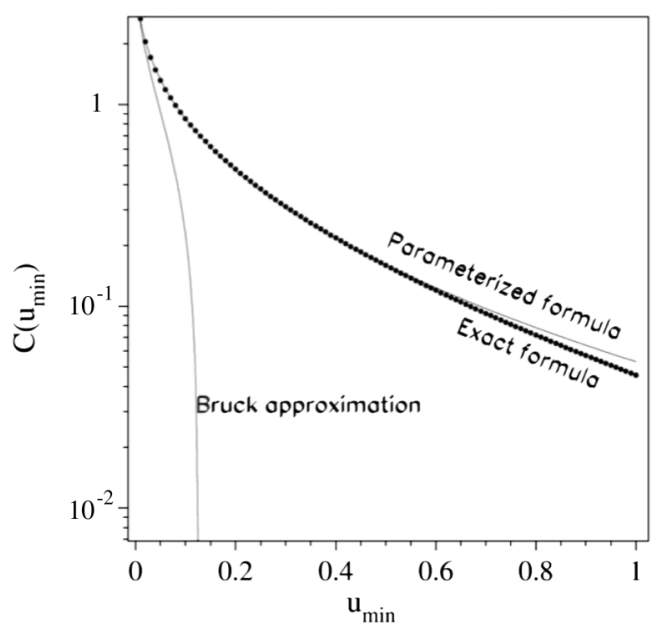

FIG. 1. Comparison between exact formula of $C\left(u_{\min }\right)$ as in formula (5) and its parametrization. The numerical fit starts to diverge from the exact formula only at $u_{\min }$ values larger than 0.7 Approximation can be used only for small $u_{\min }$.

storage rings are very much affected by it, because of the relatively low energy with high bunch current and low emittance. Lifetime and backgrounds are the main quantities affected by it. Consequently, DAфNE but also the new $B$ factories SuperKEKB [4] and SuperB [5] pay close attention to his issue.

The Touschek loss rate depends quadratically on the beam current up to a given bunch current $I_{\text {bunch }}$, that is, as far as $I_{\text {bunch }}$ is small enough to assume bunch length independent from bunch current; on the other hand, above a certain threshold, due to bunch lengthening the bunch length increases with bunch current as $I^{1 / 3}$, so the dependence of loss rate on current is $\dot{N} \sim I^{5 / 3}$. However, numerical simulations can be performed for different $I_{\text {bunch }}$ using the correct bunch length.

Another parameter closely related to bunch density is the beam coupling $\kappa=\varepsilon_{y} / \varepsilon_{x}$. The Touschek lifetime decreases as the square root of $\kappa$, and consequently, the loss rate as $\dot{N} \sim 1 / \sqrt{\kappa}$.

Numerical simulations are useful to determine-for a given machine-what are the loss rates along the ring and especially at the interaction region (IR), where are the loss points of the Touschek scattered particles to study the proper radiation shielding for the experiments and to find the right longitudinal position and shape of collimators.

Nevertheless, it is worth mentioning that in a real machine operation, after these careful simulation studies, lifetime and backgrounds are then further optimized with optics adjustments, i.e., orbit optimization, sextupoles, and octupoles tuning, and an increase of dynamic aperture with better values of $\beta$ functions on sextupoles.

The Touschek effect is simulated using a Monte Carlo technique. Section II describes in detail this simulation code, schematically shown in Fig. 2.

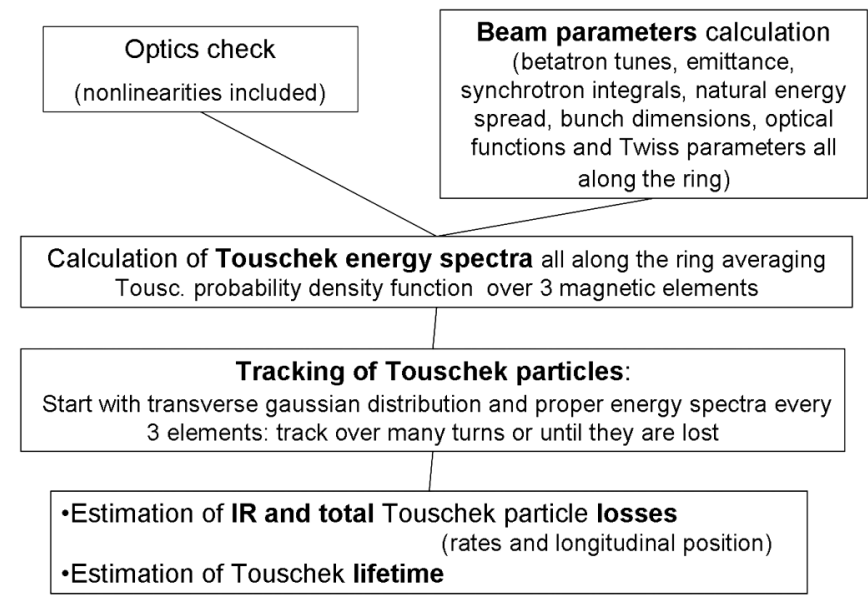

FIG. 2. Flowchart of the Monte Carlo simulation code STAR.

\section{MONTE CARLO SIMULATION}

Pioneer works on the Touschek effect simulation using Monte Carlo methods have been performed by [6]. Simulations of the Touschek effects have been performed since the beginning of the DAфNE project [7]. Since then the initial tool has been improved substantially, allowing evaluation of particle losses all along the ring and lifetime at the same time, inclusion of nonlinear terms, multiturn tracking, fast and full (GEANT3 [8]) scraper simulation, and interface with detector simulations. All of these features are implemented in STAR (Simulation of Touschek pARticles).

All of these features are obtained with the use of Monte Carlo integration methods. The Touschek scattering probability is well known in terms of the energy deviation; the particles' loss probability (i.e. the $\varepsilon=\Delta E / E$ threshold above which the particle is lost) for a beam at a given energy depends on many parameters: scattering longitudinal position along the ring, transverse position and divergence, bunch length and transverse sizes, optical functions including nonlinear terms but also on actual beam pipe geometry, including scrapers. The dependencies on the many machine parameters are accounted by the Monte Carlo method. Macroparticles representing a population of bunch particles in the transverse phase- space are subject to Touschek scattering with a weight proportional to the number of particles represented and to a scattering probability as in Eq. (4). The macroparticles are then tracked along the ring and losses are easily evaluated. The Monte Carlo is described in the following paragraph.

The input file defines the main beam parameters, while the lattice input deck is derived by the Twiss output of the MAD program [9]. $\beta$ functions and dispersion are calculated by multiplication of the first order matrices of all the magnetic elements. The optical functions are used for the horizontal and vertical beam size evaluation in each longitudinal position $s$. Different kinds of bunch distributions can be assumed. In the study presented here a transverse 
Gaussian distribution is considered. With this assumption, $\sigma_{x}$ and $\sigma_{y}$ are given by the standard definitions: $\sigma_{x}(s)=$ $\sqrt{\varepsilon_{x} \beta_{x}(s)+\left(D_{x}(s) d E / E\right)^{2}}$ and $\sigma_{y}(s)=\sqrt{\varepsilon_{y} \beta_{y}(s)}$, where $\varepsilon_{x}$ and $\varepsilon_{y}$ are the horizontal and the vertical emittance. Horizontal emittance is either derived from the calculation of the synchrotron radiation integrals or given as an input value. Vertical emittance is derived from the horizontal one and the coupling, given as an input value.

A given number of macroparticles NPART is extracted randomly from this bunch distribution, while the actual number of tracking particles is given by

$$
2 \cdot N P A R T \cdot N E L E M / 3,
$$

where NELEM is the number of magnetic elements in the lattice. For each macroparticle extracted with a given energy deviation $d E / E$, there is another one with opposite sign $-d E / E$, and the macroparticles are extracted every 3 magnetic elements.

As an example of relation (6) for the DApNE collider, $10^{6}$ macroparticles are tracked with NPART $=1220$, as the elements of the lattice are 1230. For the SuperB collider, $10^{6}$ macroparticles are tracked with $N P A R T=650$, as the ring is much longer (circumference is $1.2 \mathrm{~km}$ compared to DAфNE of $98 \mathrm{~m}$ ), but lattice is described by about twice the magnetic elements.

Each macroparticle has a different position in the transverse phase space $\left(x, x^{\prime}\right)$ and $\left(y, y^{\prime}\right)$ and a particular energy deviation $d E / E$, which is linearly random extracted within a given energy interval, set by a minimum value below which no particles are lost and a maximum one given by the rf acceptance.

The energy dependence of the Touschek scattering probability density function for particles with relative energy deviation $\varepsilon=\Delta E / E$ is very nonlinear:

$$
1 / \tau=a|\varepsilon|^{b},
$$

where $a$ and $b$ are two parameters that depend on the optical functions [10]. This behavior is determined by fitting with a linear extrapolation function (4) that, in turn, depends on beam size. Properly slicing all the elements in the lattice and evaluating there the transverse beam size, we obtain a good estimate of the Touschek probability density function for a given lattice. As evidenced by dedicated simulation studies, a good accuracy is obtained also in regions where the optical functions change rapidly by dividing one magnetic element in ten slices.

With this approach, the number of particles with a $d E / E$ that have been scattered within the ten slices are given by the Touschek probability density function evaluated for that energy offset, multiplied by $d E / E$ and by the normalization factor to the number of particles per bunch. Each macroparticle has a weight that gives directly the probability of being lost in $\mathrm{Hz}$, for the nominal bunch current.
These macroparticles are then tracked from their scattering point all along the ring for a few machine turns, or until they are lost either by for exceeding the rf acceptance or for hitting the physical aperture, or stopped by the scrapers. In this second case, the particle's transverse position and divergence is recorded all the way long. Figure 3(a) shows the DAфNE lattice for the KLOE-2 run in the crab-waist scheme [11], Fig. 3(b) shows the $H$-invariant [1] and the horizontal dispersion, and Fig. 3(c) shows the Touschek generation points along the ring of the particles eventually lost, from which the correlation with $H$-invariant function results clear. This is the present DAфNE configuration. Details are given in Sec. IV.

The particles' tracking is performed for the two transverse dimensions without synchrotron oscillations. Radio-frequency acceptance is calculated from input parameters ( $\mathrm{rf}$ cavity voltage, momentum compaction, harmonic number, beam energy spread) and energy loss

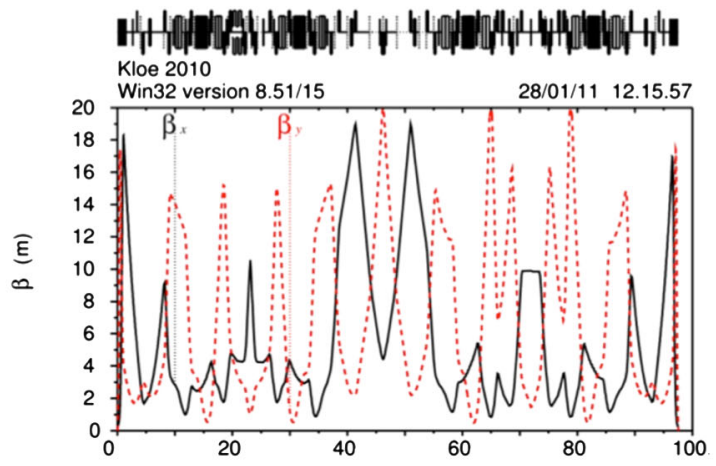

(b)

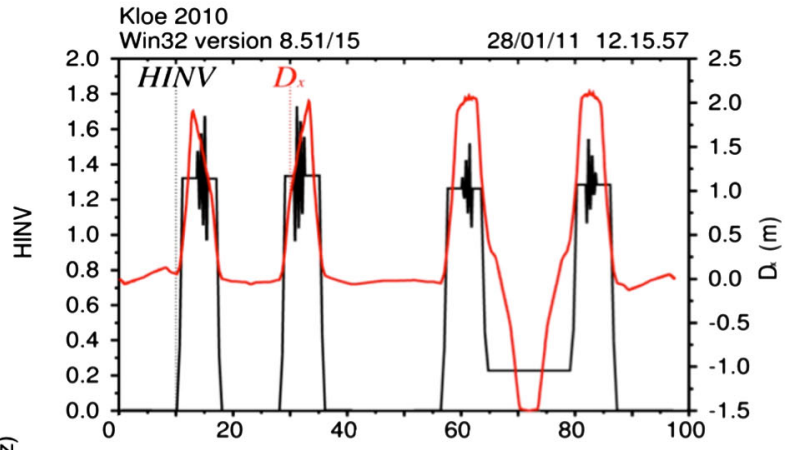

(c)

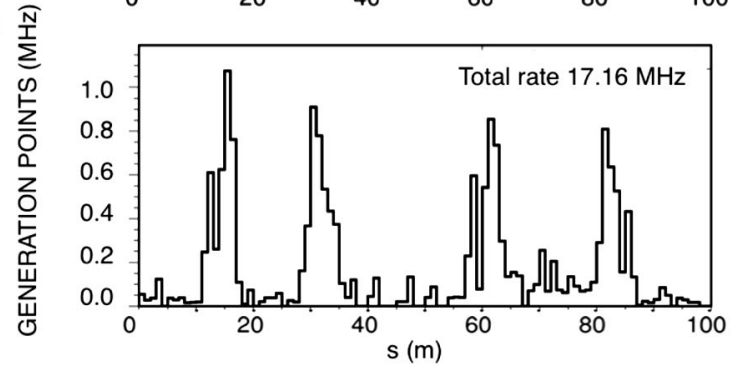

FIG. 3. Present crab-waist optics for the KLOE-2 run, the interaction point is at $s=0 \mathrm{~m}$; (a) $\beta$ functions; (b) $\mathrm{H}$-invariant and horizontal dispersion (black and red curves); (c) corresponding locations of Touschek scattering occurrence of particles that are eventually lost along the ring. 
per turn. Once per turn the macroparticle's energy deviation is compared to rf acceptance and, if larger, it is lost. This approximation is good enough to estimate the loss rates caused by exceeding the rf acceptance, even if their longitudinal loss position is not determined. On the contrary, with this approach we have the advantage to estimate this loss source without introducing radiation and damping, and consequently, without tracking scattered particles for few damping times.

Sextupoles and higher order multipolar terms are included in the particles' tracking. Nonlinear terms, such as sextupoles and octupoles but also higher order ones, are treated as a thin lens placed between two drifts of half their length. The simulation code evaluates the energy acceptance by giving as output the Touschek loss probability as a function of the energy deviation $d E / E$ (see plot in Fig. 4 as an example, referred to the low energy ring of SuperB). This evaluation can be compared with dynamical aperture simulations from dedicated codes, and checked by measurements. In this approach, the dynamical aperture is intrinsically taken into account and not imposed as input. We point out that energy acceptance estimated by STAR has always resulted in good agreement with the one calculated with MAD. This method is intrinsically very sensitive to the physical aperture, especially at the IR, where the beamstay-clear is relatively smaller with respect to the rest of the ring.

Tracking with nonlinear terms is important especially for machines with long transverse damping times, as resonances that may be excited by nonlinearities can have an effect on the beams for a long time; DAфNE is an example, due to its low energy and small circumference. Table I reports a complete list of DAфNE present parameters.

The simulation code gives a direct evaluation of the lifetime by counting the particle losses along the ring for a number of machine turns sufficient to give steady results,

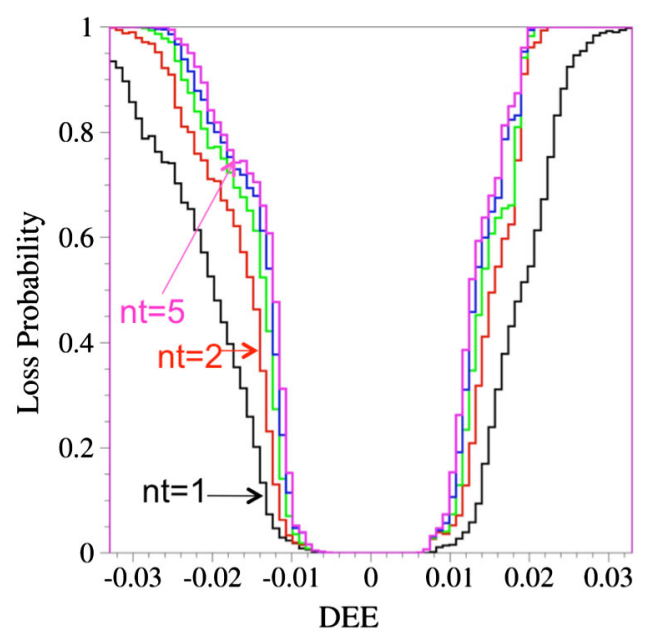

FIG. 4. Loss probability for Touschek particles as a function of their energy deviation $d E / E$, each color is for a machine turn $(n t)$, for the low energy ring SuperB lattice.
TABLE I. Main parameters of the upgraded DAфNE accelerator for the KLOE-2 run.

\begin{tabular}{ll}
\hline \hline DAфNE parameter & Value \\
\hline Maximum beam energy & $510 \mathrm{MeV}$ \\
Circumference & $97.59 \mathrm{~m}$ \\
rf frequency & $368 \mathrm{MHz}$ \\
Horizontal/vertical emittance & $240 \times 10^{-9} / 2.4 \times 10^{-9} \pi \mathrm{m} \mathrm{rad}$ \\
Energy spread & $0.4 \times 10^{-3}$ \\
Full crossing angle & $50 \mathrm{mrad}$ \\
Bunch length & $1.43 \mathrm{~cm}$ at $I_{\text {bunch }}=10 \mathrm{~mA}$ \\
$\#$ particles per bunch & $2.1 \times 10^{10}$ at $I_{\text {bunch }}=10 \mathrm{~mA}$ \\
$\beta_{x} / \beta_{y} \beta$ functions at IP $(H / V)$ & $0.25 \mathrm{~m} / 9 \times 10^{-3} \mathrm{~m}$ \\
Time between collisions & $2.7 \mathrm{~ns}$ \\
Beam rigidity $B \rho$ & $1.70 \mathrm{~T} \mathrm{~m}$ \\
Energy loss per turn & $9.11 \mathrm{keV}$ \\
\hline \hline
\end{tabular}

evaluated from formula (3). In addition, the particle losses close to the interaction point (IP) can be used as primaries for tracking with GEANT the secondaries into the detector allowing the evaluation of the background counting rates and detailed studies of background properties, namely, spatial distribution and energy spectra. Several comparisons have been performed between simulations for DAфNE and KLOE data showing a good qualitative agreement [12-14]. Recently, this study has been carried on also for the present optics; Fig. 5 shows the distributions and trajectories of the primary Touschek particles hitting the pipe for this configuration.

Some approximations have been done for the simulation of the particles tracking: the magnets, as well as the beam, are assumed perfectly aligned and centered along the beam orbit. The real machine is unavoidably more complicated, and the dynamic aperture may result not as well optimized as in simulation. Nevertheless, the approach of the Monte Carlo simulation gives a realistic estimate

(a)

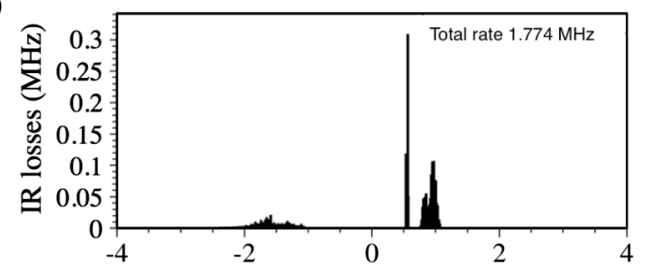

(b)

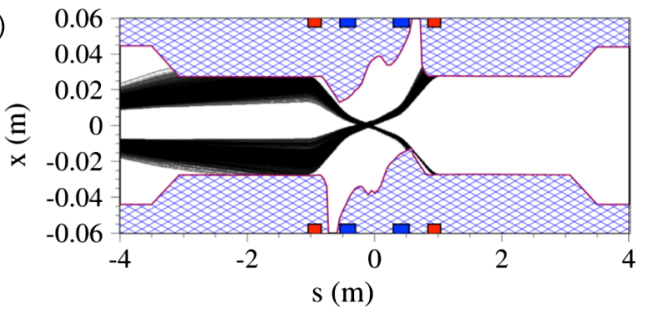

FIG. 5. (a) IR loss particles distribution for the KLOE crabwaist optics, with scrapers at their experimental set. (b) Trajectories with hit positions on the physical aperture that the particles actually see. 
(a)

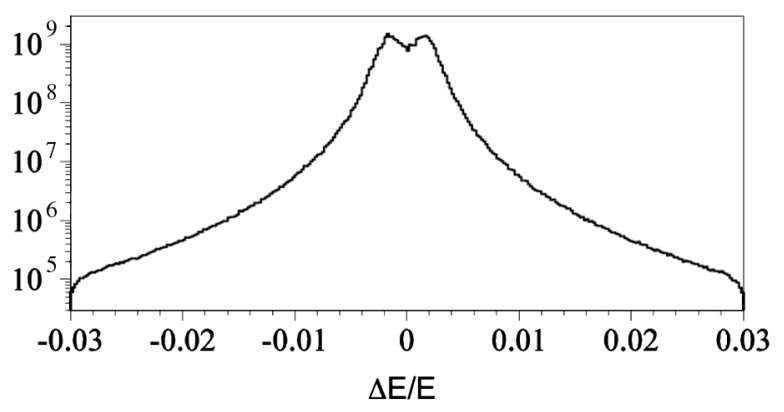

(b)

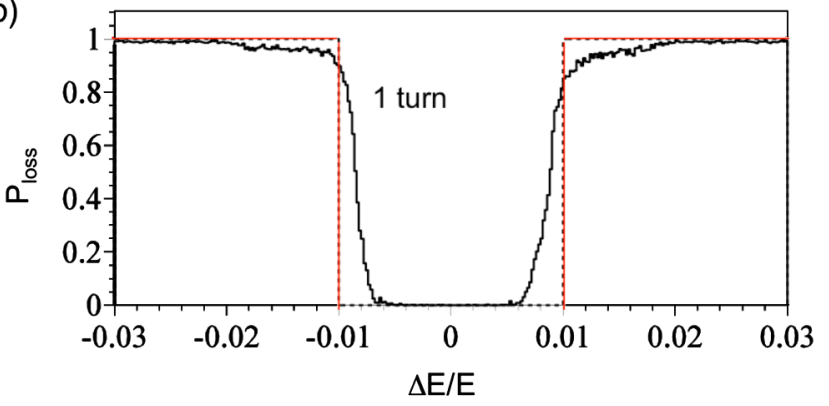

FIG. 6. SuperB low energy ring case: (a) Touschek probability density function as a function of energy deviation, i.e., generated Touschek particles per second all over the ring. (b) Comparison between the probability of particle loss due to Touschek scattering versus their energy deviation resulting from Bruck's formula assuming an energy acceptance of $1 \%$ and from STAR.

of the Touschek effect, especially if compared to the evaluation of the formula based on the assumption on the energy acceptance. Figure 6(a) shows the Touschek probability density function as a function of particles energy deviation for the SuperB low energy ring [15], in which the lower energy deviation correspondence to a higher Touschek scattering probability results clearly. Figure 6(b) shows the Touschek particles loss probability as a function of the correspondent energy deviation for this particular lattice (black curve), compared to the loss probability when $1 \%$ of energy acceptance is assumed (red curve), resulting as a step function, from which the better accuracy given by the Monte Carlo is inferred and explained by the very nonlinear behavior especially at low energy deviations. This leads to the need of a Monte Carlo approach that evaluates Touschek effect along the ring.

The application of the Monte Carlo to the DAфNE collider is discussed in the next section, where the major results that have been obtained in understanding the Touschek background during these years of machine runs are summarized.

\section{DAФNE EXPERIENCE}

At the beginning of all the experiments, DAфNE suffered from large induced background, which has always been mainly Touschek. Many efforts have been put in its reduction since the first experiments' data taking [16], which have been chronologically: KLOE (20012002, 2004-2006), DEAR (2002), FINUDA (2003, 2007), SIDDHARTA (2009), and now KLOE-2 (since 2010). The machine in the first design had two IPs symmetrical at opposite sides, even if two experiments never took data simultaneously. At IP1 was positioned KLOE, at IP2 DEAR, and later FINUDA. For the crab-waist scheme test major upgrades have been done to the machine, including the separation of the two vacuum chambers at IP2, leaving only IP1 as a low- $\beta$ region for collisions. SIDDHARTA is the experiment that took data during the crab-waist scheme test and presently KLOE-2 is running. Dedicated studies on the two existent collimators efficiencies suggested improvements in the simulation code, such as multiturn tracking including nonlinearities in the optics and, most importantly, the insertion of three additional collimators, which have been placed in optimized longitudinal positions with a shape optimized by simulations. Dedicated simulations studies suggested adjustments of optical parameters and improvement of detector shielding.

Since the machine design [17] to protect the detectors of the experiments from off-momentum particles, two horizontal scrapers were positioned at about $7.0 \mathrm{~m}$ from the two IPs. Until December 2000 they consisted of rectangular tungsten blocks of $35 \mathrm{~mm}$ thickness, which corresponds to 10 radiation lengths. They were shielded by a $150 \mu \mathrm{m}$ thick copper strip to minimize the discontinuity in the vacuum chamber.

In an effort to understand the unveiled inefficiency of the used scrapers, simulation of Touschek particles in the accelerator as well as tracking $510 \mathrm{MeV}$ electrons through the scraper blocks have been performed. Simulations of edge effects of the scrapers have been performed with STAR and GEANT3. It has been found that with the rectangular shape most of the particles are scattered by the thin copper layer above the tungsten, instead of being absorbed, thereby producing additional background to the experiments. These calculations resulted in the proposal of new modified scrapers, which were constructed and installed in the DAфNE rings during January 2001. The inner surface of the new scraper block has been divided into two flat parts. A first $10 \mathrm{~mm}$ long section has a slope of $100 \mathrm{mrad}$ towards the beam, in order to increase the impinging angle into the block for most particles. This is followed by a second section of $45 \mathrm{~mm}$ length, which slopes by $10 \mathrm{mrad}$ in the opposite direction to avoid forward scattering of electrons back into the beam pipe. A vertical slit has been introduced into the copper shield to ensure that all incident particles only see the tungsten absorber. The total scraper thickness of now $55 \mathrm{~mm}$ (about 16 radiation lengths) is reducing the punch-through probability of $500 \mathrm{MeV}$ electrons to below $10^{-6}$. The behavior of the new scraper is shown in Fig. 7 (full red line). The external 
jaw reduces the background by a factor 2.9 at a distance of $\approx 20 \mathrm{~mm}$ from the chamber axis. No background reduction is found when moving in the internal jaw (full line), however, the previously observed strong increase is no longer present (dashed line), indicating an improved stopping efficiency of the scraper blocks.

The two rates from the KLOE [18] electromagnetic calorimeters in the electron forward region (ECM2 and ECM4) integrate the signals over the four innermost sectors of the west and east forward calorimeters each. In order to compare measurements with different beam parameters, the calorimeter rates are given per $1 \mathrm{~mA}$ bunch and are scaled for a ratio of the vertical to horizontal beam size measured at the location of the synchrotron light monitor of 0.1. Rates of the two forward calorimeters in KLOE have been taken as a function of the opening of the horizontal scrapers upstream the IR for these measurements.

To obtain a further background reduction, three additional scrapers have been installed in high $\beta_{x}$ and dispersion positions, along the arcs of each ring in January 2002. The efficiency of these scrapers has been simulated by tracking Touschek scattered particles from their origin in the arcs into the KLOE IR and it has been compared to measurements. The electromagnetic calorimeter rates in the electron forward region counter have been evaluated by using the KLOE detector simulation program GEANFI [19]. A parametrization of this acceptance as a function of the relevant particle informations $\left(s, x\right.$ and $\left.x^{\prime}\right)$ has been included in STAR, allowing a comparison between the measured and the calculated calorimeter rates as a function of the scraper openings. A satisfactory qualitative agreement has been found [20].

A factor 1.6 in the scraper efficiency is gained due to the fact that it can be inserted closer to the center of the pipe.

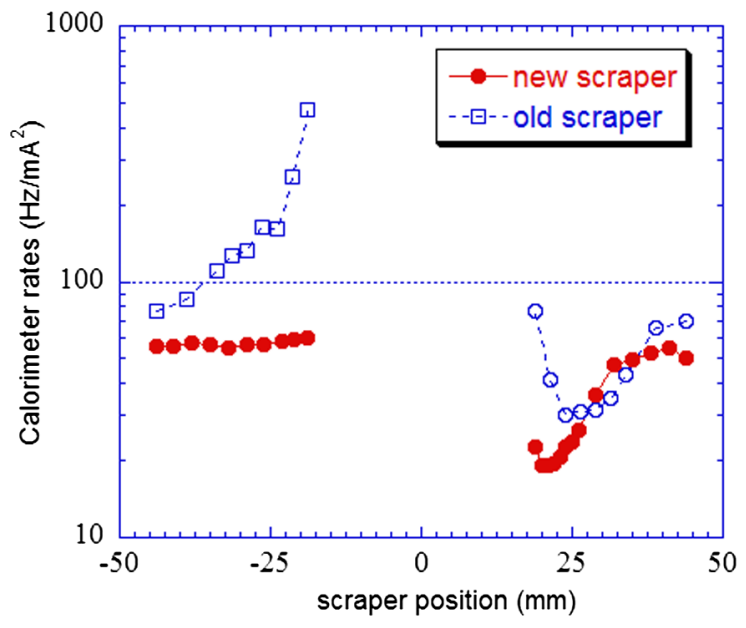

FIG. 7. Scan of the normalized background rate versus the position of the inner scraper edge. The scraper openings are measured from the central beam axis.
The added scrapers stop particles that would be just deviated by the IR scraper and eventually lost at the experiment. In that KLOE running configuration the overall effectiveness of the scrapers was about a factor of 3 for the positron beam and $\simeq 7$ for the electron one. However, with the scrapers inserted, the contributions of the two beams to the end cap rates were about the same.

Figure 8 shows also the corresponding calculated rate, evaluated by tracking Touschek scattered particles from their origin in the arcs into the KLOE detector. The end cap acceptance has been taken into account by means of full detector simulation including the geometrical details of the IR. There is a qualitative agreement between measurement and simulation.

An important feature that has been implemented in the simulation code is that all magnetic elements have been considered, including sextupoles and octupolar components in the wigglers. In fact, beam losses at the experiments are very sensitive to machine nonlinearities, i.e., sextupoles settings [21]. Careful tuning of sextupoles can reduce the background rates at the experiments, in agreement with simulations, as appears from Fig. 9. At DAфNE each sextupole is powered independently, hence enabling their careful tuning. A comparison between the expected background reduction and the measured one has been performed by varying four sextupoles close to their setting values. The comparison has been performed on the KLOE calorimeter rates in the electron forward region, where its efficiency has been evaluated with full simulation (GEANT3) [22] and included in the code. An example is shown in Fig. 9 where the KLOE calorimeter rates are measured for different sextupole strengths. These measured rates are properly normalized to the same initial beam current and same beam transverse size. When the current of SXPPL102 goes from 0 to $30 \mathrm{~A}$ the KLOE calorimeter

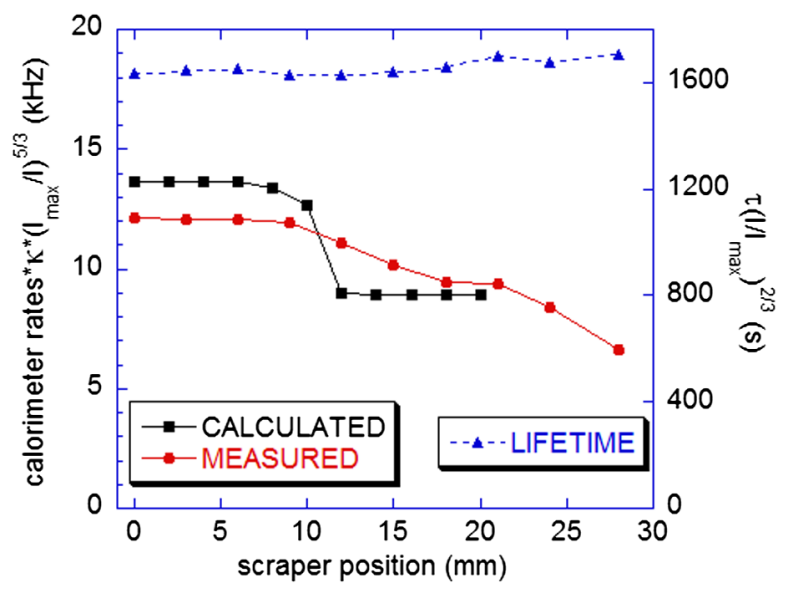

FIG. 8. Scan of the background rate in the KLOE forward calorimeter versus position of the internal jaw of the scraper SCHPL110: measured (calculated) normalized rate in red dots (black squares) and normalized measured lifetime in blue triangles. 


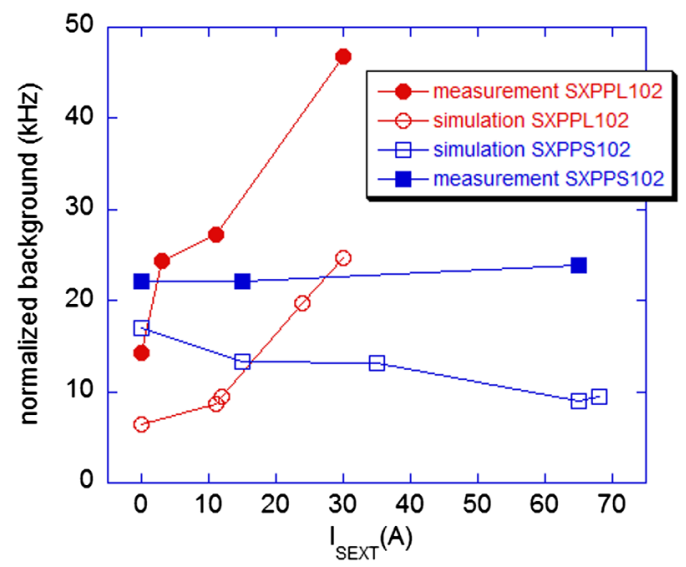

FIG. 9. Normalized background $(\mathrm{kHz})$ measured by the KLOE EmC as a function of the strength of two sextupoles (full markers), compared to calculations (empty markers).

rates increase by a factor 3.3 (full red dots), in agreement with that expected from simulation (empty red dots).

A similar analysis is performed with sextupole SXPPS102. Its effect on measured calorimeter rates is close to 0 (see full blue squares in Fig. 9), in agreement with simulation (empty blue squares). The overall difference between the measured and the calculated background rate is consistent with the uncertainty in the tracking of the loss particles from the beam pipe to the calorimeter through the complex geometry of low- $\beta$ magnets, electronics, and services.

During the DEAR run (March-April 2002), the machine background has been reduced both by properly shielding the detectors and by an optics change in the IR, consisting in reducing the beam envelope where the particles are expected to be lost, i.e., by reducing the $\beta_{x}$ function. This optics change allowed for a background reduction to the experiment of a factor 3.3.
The machine IR design was originally a triplet, with a focusing-defocusing-focusing low- $\beta$ quadrupole scheme, with the nominal $\beta_{x}$ value at IP of $4.5 \mathrm{~m}$. The main losses due to Touschek scattering are found from simulation to be placed in the region between the last focusing quadrupole and the IP [see lower plot of Fig. 10(a) for the trajectories and upper one for the distribution of loss rates]. A reduction of these particle losses in this region has been obtained by reducing the beam envelope size, which, in turn, has been obtained by reducing $\beta_{x}(s)$ [plots of Fig. 10(b)]. $\beta_{x}$ at IP2 has been reduced by a factor 2.8 (from $\approx 4.5 \mathrm{~m}$ to $\approx 1.6 \mathrm{~m}$ ) and consequently at the focusing quadrupole closer to the IP2, allowing at the same time the increase of the luminosity. With this change the particles losses are moved downstream the IP hitting the aperture at the first focusing quadrupole, as indicated in Fig. 10(b). These particles have been easily shielded.

The experience gained with DEAR has been useful for the design of a new IR for KLOE, installed on DAфNE at the end of 2002, which provided a lower $\beta_{x}$ at the focusing quadrupole close to IP1 and shielding behind the other quadrupole farther from IP1, lowering the backgrounds (see Fig. 11) while enhancing the luminosity. With the new interaction region design, including detector shieldings and scrapers, a reduction of about 1 order of magnitude has been obtained for the luminosity normalized detector background rate. In particular, the background closest to the IP inducing in the KLOE tracking system about $200 \mathrm{~Hz}$ protons from photoproduction has been totally eliminated. Details are given in the following.

The Touschek particles lost at the KLOE IR have been fully simulated in the detector allowing the evaluation of the background counting rates and detailed studies of their properties, namely, spatial distribution and energy spectra. The results of the Touschek simulation showed a huge background rate entering in the KLOE region. Most of

(a) DEAR IR: triplet FDF scheme

(b) DEAR IR: doublet FD scheme
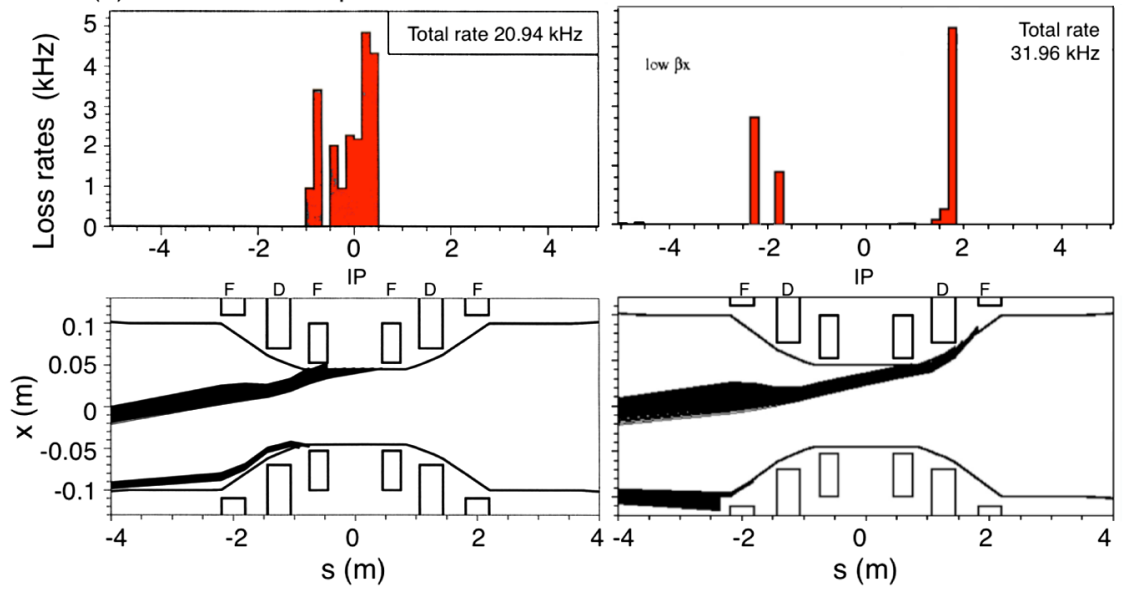

FIG. 10. Upper and lower plots show distribution and trajectories of Touschek particles lost at the DEAR IR for the two low- $\beta$ configurations: (a) focusing-defocusing-focusing quadrupoles, for which the lowest $\beta_{x}$ value at IP is $4 \mathrm{~m}$; (b) focusing-defocusing quadrupoles, for which the lowest $\beta_{x}$ value at IP is $1.5 \mathrm{~m}$. 

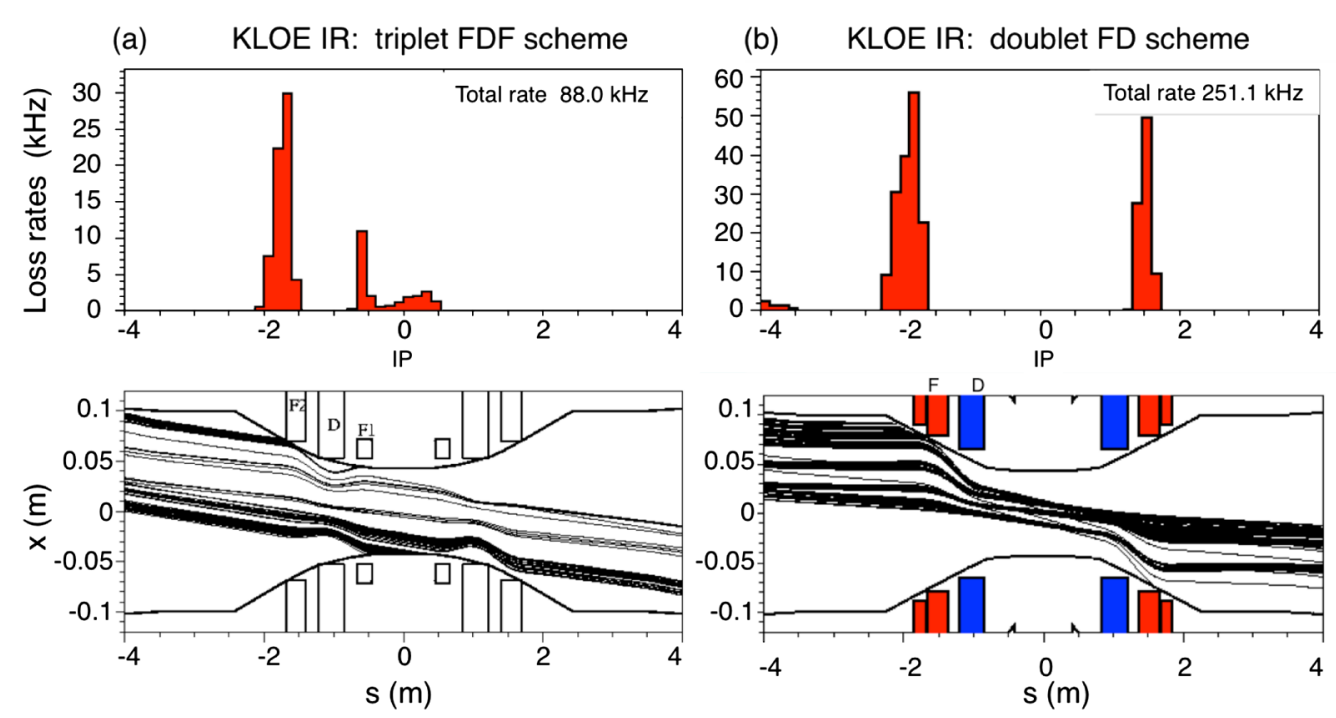

FIG. 11. Upper and lower plots show distribution and trajectories of Touschek particles lost at the KLOE IR for the two low- $\beta$ configurations: (a) focusing-defocusing-focusing quadrupoles; (b) focusing-defocusing quadrupoles, successfully used for KLOE masks design.

this background was expected to hit the first focusing low- $\beta$ quadrupole, being the subject of the comparison with the KLOE data [the region around $|z| \sim 0.5 \mathrm{~m}$ in Fig. 12(a)]. Since in this case the products of the primary interactions induced by the Touschek particles were not shielded, they could be measured precisely with the KLOE detector. A dedicated run on June 2001 has been analyzed and compared to the expected background. Figure 12(b) shows the expected hits distribution around $|z| \sim 0.5$; the two peaks are around the quadrupole regions, in the external side of the machine. The KLOE analysis results show the evidence for photoproduction induced by machine background. A toy simulation (see [12] for details) has been used to evaluate, starting from the Monte Carlo output, the rate of the photoproduction induced by background and of the corresponding interaction point distribution. The expected rate is about $280 \mathrm{~Hz}$ in agreement with the rate observed by KLOE, which is about $300 \mathrm{~Hz}$. Figure 12(a) shows the distribution of the point of closest approach to the beam line of the tracks from KLOE data to be compared with that expected from background simulations [Fig. 12(b)]. The peak in Fig. 12(a) around $s=0$ at a much smaller intensity is due to $\mathrm{e}^{+} \mathrm{e}^{-}$collisions which was difficult to extract at trigger level being based on calorimetry. The asymmetry between electron and positron beam background is observed in experimental data and reproduced by simulations, as well as the strong asymmetry of the background distribution on the machine plane (the particle losses are measured and predicted mostly in the external region to the ring). The new interaction region
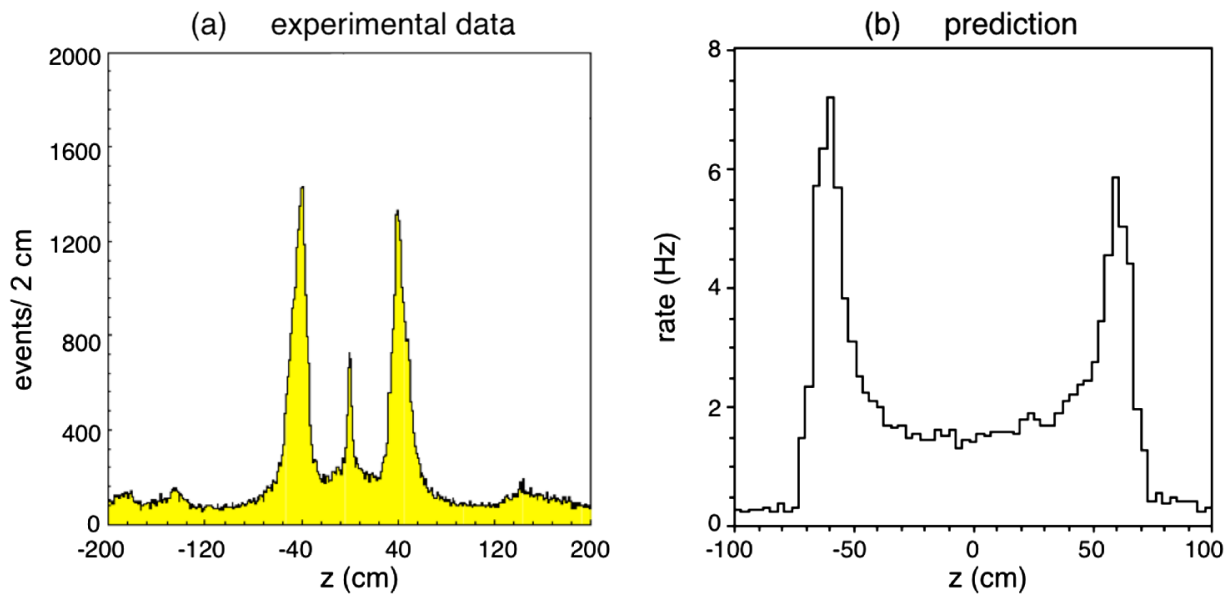

FIG. 12. (a) High rate of localized monotracks (protons) in KLOE until 2001 understood as photoproduction $e p(n) \rightarrow \Lambda e \rightarrow$ $p \pi^{0}\left(\pi^{-}\right) e$, induced by Touschek particles hitting the beam pipe support; (b) prediction from full simulation of Touschek particles into detector. 
TABLE II. Comparison of main beam parameters for KLOE (2006), SIDDHARTA (2008-2009), and present KLOE-2 runs for luminosity and lifetime and backgrounds.

\begin{tabular}{lccc}
\hline \hline & $\begin{array}{c}\text { Standard } \\
\text { scheme } \\
\text { Parameters }\end{array}$ & $\begin{array}{c}\text { Crab-waist } \\
\text { scheme }\end{array}$ & $\begin{array}{c}\text { Crab-waist } \\
\text { scheme }\end{array}$ \\
\hline$I(\mathrm{~mA} /$ bunch) & 13 & 10 & 10 \\
$\theta_{\text {cross }} / 2(\mathrm{mrad})$ & 12.5 & 25 & 25 \\
$\beta_{x} *(\mathrm{~m})$ & 1.5 & 0.27 & 0.25 \\
$\beta_{y} *(\mathrm{~mm})$ & 18 & 9 & 9 \\
$\sigma_{z}(\mathrm{~cm})$ & 2.5 & 1.6 & 1.4 \\
$\varepsilon_{x}(\mathrm{~mm} \mathrm{mrad})$ & 0.3 & 0.26 & 0.26 \\
\hline \hline
\end{tabular}

design reduces this background component to a negligible value.

\section{DAФNE WITH THE CRAB-WAIST SCHEME}

The innovative crab-waist collision scheme has been implemented at DAфNE with a new KLOE-2 run [23] after its first successful test during the SIDDHARTA run [24].

With this configuration transverse beam sizes are small, especially at the IR, and the Touschek effect requires special attention, both for the lifetime and the machine induced backgrounds. The features of this novel scheme that affect the Touschek scattering effect are the smaller emittance and the smaller transverse beam sizes, especially at the IP (see Table II for comparisons with different optics). Touschek lifetime is short and particle losses that induce backgrounds into the experiments are high, as we verified during the crab-waist scheme test with the SIDDHARTA run, together with a good agreement with expectations $[25,26]$. However, remedies to counteract this effect can be undertaken and at the same time take profit of luminosity enhancement as, for example, implementing proper shielding to decrease the impact of backgrounds on the detector performance.

Dedicated studies for data and numerical simulation comparisons are carried out for the new KLOE-2 run, as done for the previous experiments. The DAфNE optical model has been tuned to keep the effects of Touschek scattering under control with a trade-off between critical parameters, following the indications given by simulations. New simulation studies have been performed with the actual beam parameters (listed in the last column of Table II) and the present lattice for the new KLOE-2 run. Comparisons between measured and calculated Touschek lifetime have been performed for the electron beam, with and without scrapers, as shown in Fig. 13(a). The same experimental set of scrapers has been used for simulations. Bunch length has been varied in the simulations accordingly to bunch current, taking into account also the slight difference resulting from the measurements, with or without insertion of scrapers (Fig. 4 of [23]).

As appears from Fig. 13(a), there is a good agreement between measured and calculated lifetime with scrapers inserted (blue full dots and red full squares, respectively). On the contrary, the comparison without scrapers (blue empty dots and red empty squares, respectively) shows a disagreement of about a factor 1.9, which might be explained by a misalignment of the on-energy beam orbit that induced beam scraping in the IP2 section, as found after these measurements. In addition, measured dynamic aperture was not as large as the expected one, both from MAD and from STAR.

Summarizing, numerical simulations indicate a Touschek lifetime of $\tau=620 \mathrm{~s}$ for a $10 \mathrm{~mA}$ bunch current, $0.4 \%$ beam coupling, with a calculated set of sextupoles that optimizes dynamic aperture and with the same experimental set of scrapers. This set is: scraper upstream IP at $-14 /+11 \sigma_{x}$, scraper close at the opposite side of IP at $\pm 9 \sigma_{x}, \mathrm{SCHEL} 110$ at $+36 \sigma_{x}$, and SCHES101 at $21 \sigma_{x}$. (a)

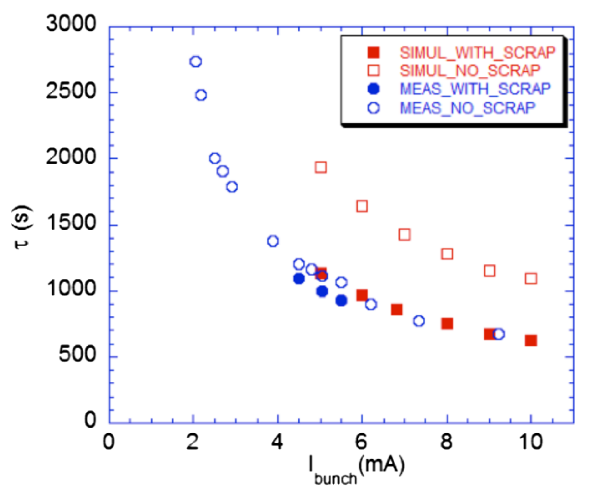

(b)

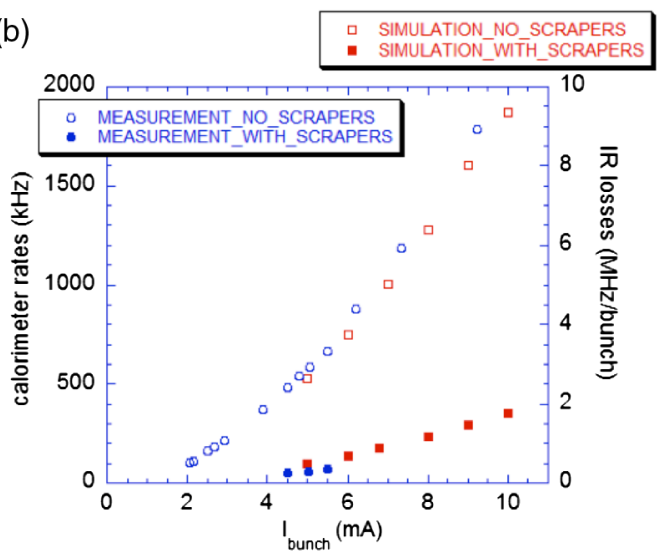

FIG. 13. Measured (blue dots) and predicted (red squares) electron beam (a) lifetime and (b) losses versus bunch current, with $\kappa=0.4 \%$. 


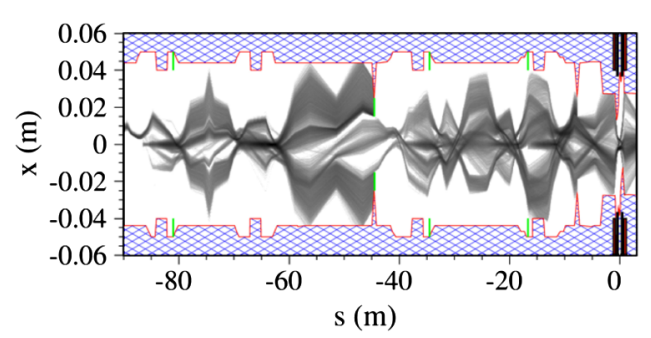

FIG. 14. Trajectories of Touschek particles lost along the ring, in green the horizontal scrapers placed at the experimental set, red line is the physical aperture.

For the KLOE- 2 run only the scrapers upstream the IP and the one close at the old IP2 have been moved, according to the new lattice. The first one has been placed at about $8 \mathrm{~m}$ upstream the IP, while the second one at a high $\beta_{x}$, downstream the old IP2. In addition, the beam-stay-clear at these two scrapers has been reduced to intercept Touschek scattered particles that would be lost at the IR: the IP1 scraper is at $18 \sigma_{x}$ from the center of the vacuum center already with no jaws inserted, the second one is at $14 \sigma_{x}$. Figure 13(b) shows the comparison of the electromagnetic calorimeter rates in the electron forward region and the calculated IR losses within $4 \mathrm{~m}$ from the IP. The two quantities are in different units, since the left $y$ axis stands for the experimental count rate and the right one for simulated particle loss rate, and they cannot be compared directly. However, Fig. 13(b) tells us that they scale equally with current, even if the effectiveness of scrapers is somewhat different: experimentally calorimeter rates (blue empty and full dots) are reduced by a factor 9.5 , while IR losses, for the same scrapers set, only by a factor 5.4 (red empty and full squares).

A direct comparison of calorimeter rates and IR particle losses is possible only after a full simulation of showers into the calorimeter. IR distributions of the Touschek particle losses are tracked from the beam pipe into the KLOE calorimeter for direct comparison of measured and expected background rates. In addition, these studies are carried out with the same software tools used for the SuperB design, allowing a direct validation test of this approach.

An example of the trajectories of Touschek particles that are lost along the ring is shown in Fig. 14.

Adiabatic adjustments of the optical parameters, sextupoles tuning, and orbit optimization, especially at the IR, are important knobs that improve backgrounds during data taking.

\section{SUMMARY}

The Touschek scattering is one of the main effects in low emittance rings that determine the beam lifetime and the particle losses. In this paper we have described in detail the STAR simulation code, based on the
Monte Carlo technique. This tool has allowed the minimization of backgrounds induced in the detectors at DAфNE, thanks to dedicated studies of position and shape of scrapers and detector shieldings, interaction region optics design, and relevance of nonlinear effects. Corresponding data/Monte Carlo simulation comparison for each action taken has been presented, showing an overall good agreement.

During the first KLOE run, a luminosity normalized to background reduction of more than 1 order of magnitude has been obtained with a concomitant increase in luminosity from $10^{31}$ to $10^{32} \mathrm{~cm}^{-2} \mathrm{~s}^{-1}$ and only a small decrease in lifetime. With the gained experience the run with the crabwaist scheme for SIDDHARTA has been successfully carried out, while the long run for KLOE-2 is approaching.

\section{ACKNOWLEDGMENTS}

I would like to thank S. Guiducci, who has worked on this item in the first stage of the DAфNE design and commissioning. The collaboration with experiments (KLOE, DEAR, SIDDHARTA, LUMI group, and SuperB) for understanding Touschek backgrounds induced in detectors has been continuous and useful for both sides.

[1] R. H. Helm, M. J. Lee, P. L. Morton, and M. Sands, IEEE Trans. Nucl. Sci. 20, 900 (1973).

[2] H. Bruck, Accelerateurs Circulaires de Particules (Presses Universitaires de France, Paris, 1966).

[3] G. Vignola et al. (DAфNE Project Team), in Proceedings of the Particle Accelerator Conference, Washington, DC, 1993 (IEEE, New York, 1993), p. 1993.

[4] http://www-superkekb.kek.jp/index.html.

[5] SuperB Progress Reports, The Collider [http://arxiv.org/ abs/1009.6178v2].

[6] S. Khan, BESSY TB No. 177, 1993.

[7] S. Guiducci, DAфNE Technical Note L-12, 1993.

[8] R. Brun et al., CERN Program Library Long Writeup W5013 (1993).

[9] H. Grote and F. C. Iselin, Report No. CERN/SL/90-13 (AP), 1996.

[10] C. Bernardini, G. F. Corazza, G. di Giugno, G. Ghigo, J. Haissinski, P. Marin, R. Querzoli, and B. Touschek, Phys. Rev. Lett. 10, 407 (1963).

[11] M. Zobov et al., Phys. Rev. Lett. 104174801 (2010).

[12] M. Boscolo, M. Antonelli, and S. Guiducci, in Proceedings of the 8th European Particle Accelerator Conference, Paris, 2002 (EPS-IGA, Geneva, 2002), p. 1238.

[13] M. Boscolo and S. Guiducci, DAфNE Technical Note IR-10, 2001.

[14] M. Antonelli, L. Passalacqua, and B. Sciascia, KLOE Memo 260, 2001.

[15] SuperB CDR, arXiv:0709.0451.

[16] M. Boscolo, S. Bertolucci, C. Petrascu, S. Guiducci, and G. von Holtey, in Proceedings of the 2001 IEEE Particle Accelerator Conference (IEEE, Chicago, 2001), p. 2032. 
[17] S. Guiducci, in Proceedings of the 5th EPAC, Sitges (Barcelona), edited by S. Myers (Taylor \& Francis, London, 1996), p. 1365.

[18] The KLOE collaboration, Report No. LNF-93/002, 1993.

[19] F. Ambrosino et al., Nucl. Instrum. Methods Phys. Res., Sect. A 534, 403 (2004).

[20] M. Boscolo and S. Guiducci, DAфNE Technical Note IR-11, 2002.

[21] M. Boscolo, in the Proceedings of the 23rd Advanced ICFA Beam Dynamics Workshop on High Luminosity $\mathrm{e}^{+} \mathrm{e}^{-}$Colliders, 2001 (Cornell University, Ithaca, New York, 2001).
[22] M. Antonelli (private communication).

[23] C. Milardi et al. (DAфNE Commissioning Team), in Proceedings of IPAC2011, San Sebastián, Spain (EPS-AG, Spain, 2011), p. 3687.

[24] C. Milardi et al. (DAфNE Commissioning Team), in Proceedings of the 23rd Particle Accelerator Conference, Vancouver, Canada, 2009 (IEEE, Piscataway, NJ, 2009), pp. 80-84.

[25] M. Boscolo et al., Nucl. Instrum. Methods Phys. Res., Sect. A 621, 121 (2010).

[26] M. Boscolo et al., Nucl. Instrum. Methods Phys. Res., Sect. A 621, 157 (2010). 